\title{
RELATIVELY OPEN OPERATORS AND THE UBIQUITOUS CONCEPT
}

\author{
R. W. Cross
}

Abstract

\begin{abstract}
A linear operator $T: D(T) \subset X \rightarrow Y$, when $X$ and $Y$ are normed spaces, is called ubiquitously open (UO) if every infinite dimensional subspace $M$ of $D(T)$ contains another such subspace $N$ for which $T \mid N$ is open (in the relative sense). The following properties are shown to be equivalent: (i) $T$ is UO, (ii) $T$ is ubiquitously almost open, (iii) no infinite dimensional restriction of $T$ is injective and precompact, (iv) either $T$ is upper semi-Fredholm or $T$ has finite dimensional range, (v) for each infinite dimensional subspace $M$ of $D(T)$, we have $\operatorname{dim}(T \mid M)^{-1}(0)+\Delta(T \mid M)>0$. In case $T$ is closed and $X$ and $Y$ are Banach spaces, $T$ is UO if and only if $\overline{T M} \subset T \bar{M}$ for every linear subspace $M$ of $X$.
\end{abstract}

\section{Introduction}

Relatively open operators, i.e. operators for which the image of the unit ball contains a ball in the range of the operator, have certain pathologies not shared by their injective components. For instance, they are unstable under the addition of bounded finite rank operators (cf. [L2, Example 11]), composition, and even under restriction. A new class of operators which includes the relatively open operators with finite dimensional kernels, and more generally, $F_{+}$-operators enjoys a stronger property which we formulate in terms of the "ubiquitous" concept below. This latter notion was introduced in [C1] where ubiquitous continuity, precompactness and strict singularity were characterised. In the case of a closed operator between Banach spaces, ubiquitous openess is equivalent to inclusion $\overline{T M} \subset T \bar{M}$ holding for every linear subspace $M$ of the domain of $T$ (Theorem 3.11).

Let $X$ and $Y$ be normed spaces and let $L(X, Y)$ denote the class of linear transformations $T: D(T) \subset X \rightarrow Y$, where $D(T)$ is the domain of $T$. The null space and range of $T$ are denoted by $N(T)$ and $R(T)$ respectively. Write $U_{X}=\{x \in X:\|x\|<1\}$, the open unit ball of $X$. 
We call the operator $T$ open (or relatively open) if $T\left(U_{D(T)}\right) \supset \lambda U_{R(T)}$ for some $\lambda>0$. If there exists $\lambda>0$ such that $\overline{T\left(U_{D(T)}\right)} \supset \lambda U_{R(T)}$ then $T$ is called almost open. We adopt the convention that all zero operators are open and almost open, including the case where $D(T)=(0)$.

The operator $T$ is said to be partially open if there exists a finite codimensional subspace $E$ of $D(T)$ (or equivalently, of $X$ ) for which $T \mid E$ is open.

The operator $T$ is called ubiquitously open (UO) if each infinite dimensional subspace $M$ of $D(T)$ contains another such subspace $N$ for which $T \mid N$ is open. Likewise, $T$ is called ubiquitously almost open (UAO) if it has the corresponding property but with $T \mid N$ almost open instead of open. It will be seen that the UO and UAO properties are equivalent (Theorem 3.1). It is clear that in the case of an injective operator the partially open and UO properties coincide. An operator $P: D(P) \subset X \rightarrow X$ is called a projection if $P^{2}=P$. Such an operator is open, and is UO if and only if either $N(P)$ or $R(P)$ is finite dimensional (Theorem 3.3).

The operator $T$ is called an $F_{+}$-operator if there exists a finite codimensional subspace $M$ of $D(T)$ for which $T \mid M$ has a continuous inverse. For examples, properties and applications of $F_{+}$-operators see [C2], [C4], $[\mathbf{C 5}],[\mathbf{C 6}],[\mathbf{C 7}],[\mathbf{C 8}],[\mathbf{C 9}],[\mathbf{C 1 0}],[\mathbf{C 1 1}],[\mathbf{C L 2}]$, and [L2], [L3]. Theorem 3.4 states that $T$ is UO if and only if either $T$ is an $F_{+}$-operator or $R(T)$ is finite dimensional.

We note some properties enjoyed by the UO operators (Corollary 3.7): The product of two UO operators is UO whenever the product is defined. In particular, if $T$ is $\mathrm{UO}$ then so is any restriction of $T$. If $T$ is $\mathrm{UO}$ and $F$ is any finite rank operator then $T+F$ is UO.

The following terminology and notation will be used. Continuous everywhere defined operators are referred to as bounded. We write $\alpha(T)=\operatorname{dim} N(T)$, and $\bar{\beta}(T)=\operatorname{dim} Y \overline{R(T)}$. If $E$ is a linear subspace of $X$ then the operator $J_{E}^{X}$ (or simply $J_{E}$ ) is the canonical injective map of $E$ into $X$. Thus $J_{E} \in L(E, X)$. We define the adjoint of $T$ by $T^{\prime}=\left(T J_{D(T)}\right)^{\prime}$ where the right hand side is defined as in [G, II.2.2]. The minimum modulus $\gamma(T)$ of $T$ is defined [K] by $\gamma(T)=\sup \{\gamma:\|T x\| \geq \gamma d(x, N(T))$ for all $x \in D(T)\}$. Clearly $T$ is open if and only if $\gamma(T)>0$. Corollary 3.6 exhibits a uniform boundedness property enjoyed by the minimum modulus.

Let $\mathcal{I}(X)$ denote the collection of infinite dimensional subspaces of $X$. The quantities $\Delta$ and $\tau$ (cf. $[\mathbf{S}],[\mathbf{C 2}]$ ) are defined as follows. If 
$\operatorname{dim} D(T)<\infty$ then $\Delta(T)=\tau(T)=0$. Otherwise

$$
\begin{aligned}
\Delta(T) & =\sup _{M \in \mathcal{I}(D(T))} \inf _{N \in \mathcal{I}(M)}\|T \mid N\| \\
\tau(T) & =\sup _{M \in \mathcal{I}(D(T))} \inf _{m \in M} \frac{\|T m\|}{\|m\|} .
\end{aligned}
$$

The operator $T$ is called strictly singular ([D], [C2], [C4]) if there is no $M \in \mathcal{I}(D(T))$ such that $T \mid M$ has a continuous inverse (this is a generalisation of Kato's definition $[\mathbf{K}])$. The operator $T$ is strictly singular if and only if $\Delta(T)=0$, if and only if $\tau(T)=0$ [C2, 3.4 and 3.10], and partially continuous (i.e. continuous on some finite codimensional subspace) if and only if $\Delta(T)<\infty[\mathbf{C 2}, 4.3]$.

The quantity $\xi(T)$ is defined by

$$
\xi(T)=\alpha(T)+\Delta(T) .
$$

Let $X_{T}$ denote the space $D(T)$ normed by $\|x\|_{T}=\|x\|+\|T x\|(x \in$ $D(T)$ ). The graph operator $G_{T}$ (or simply $G$ ) is the canonical injection of $X_{T}$ into $X$.

\section{Preliminary Propositions}

We detail below some known results which are used in the sequel.

2.1. Let $T$ be injective and everywhere defined on a Banach space. If $T$ is bounded and if $T^{-1}$ is continuous on a subspace of finite codimension, then $T$ is an isomorphism [C1, Theorem 10].

2.2. The operator $T$ is strictly singular if and only if it is ubiquitously precompact [C1, Theorem 14], if and only if $\Delta(T)=0$ ([C2, 3.4]; see also $[\mathbf{S}, 3.2]$ ).

2.3. Let $E$ be a finite codimensional subspace of $D(T)$. Then $\Delta(T \mid E)=\Delta(T)$ and $\tau(T \mid E)=\tau(T)[\mathbf{C 2}, 2.1]$.

2.4. The operator $T$ is an $F_{+}$-operator if and only if no infinite dimensional restriction of $T$ is precompact $[\mathbf{C 2}, 2.2]$.

2.5. The product of two $F_{+}$-operators is an $F_{+}$-operator whenever the product is defined $[\mathbf{C 2}, 2.13]$. 2.1]).

2.6. The operator $T$ is open if and only if $T G_{T}$ is open (see e.g. $[\mathbf{L 1}$,

2.7. The operator $T$ is almost open if and only if $T^{\prime}$ is open (see e.g. [MS, 2.1]). 


\section{Ubiquitously open operators}

3.1. Theorem. The following statements are equivalent:

(i) $T$ is ubiquitously open.

(ii) $T$ is ubiquitously almost open.

(iii) For each $M \in \mathcal{I}(D(T))$ there exists $N \in \mathcal{I}(M)$ such that $\xi(T \mid N)>0$.

(iv) $\xi(T \mid M)>0$ for each $M \in \mathcal{I}(D(T))$.

(v) For each linear subspace $E$ of $D(T)$ such that $E \cap N(T)$ is finite dimensional, $T \mid E$ is an $F_{+}$-operator.

(vi) No infinite dimensional injective restriction of $T$ is a precompact operator.

(vii) Each infinite dimensional restriction of $T$ is partially open.

(viii) Some (every) finite codimensional restriction of $T$ is ubiquitously open.

Proof: We proceed through the following chain of implications: (i) $\Rightarrow$ (iv) $\Rightarrow$ (iii) $\Rightarrow$ (i), (i) $\Rightarrow$ (ii) $\Rightarrow$ (v) $\Rightarrow$ (vi) $\Rightarrow$ (i), (i) $\Rightarrow$ (vii) $\Rightarrow$ (i), (i) $\Rightarrow$ (viii) $\Rightarrow$ (i).

(i) $\Rightarrow$ (iv): Assume (i) and let $\xi(T \mid M)=0$ for some $M \in \mathcal{I}(D(T)$ ). Then $T \mid M$ is both injective and strictly singular by 2.2 and consequently for each $N \in \mathcal{I}(M)$ we have $\gamma(T \mid N)=0$, contradicting (i).

(iv) $\Rightarrow$ (iii): Immediate.

(iii) $\Rightarrow$ (i): Suppose $T$ is not UO. Then there exists $M \in \mathcal{I}(D(T))$ such that $\gamma(T \mid N)=0$ for $N \in \mathcal{I}(M)$. Now $M \cap N(T)$ cannot have finite codimension in $M$ (for then $T \mid M$ would have finite rank, giving $\gamma(T \mid M)>0)$. Hence by passing to an infinite dimensional subspace of $M$ if necessary, we may suppose that $T \mid M$ is injective. Then $\alpha(T \mid M)=0$ and $T \mid M$ is strictly singular, i.e. $\Delta(T \mid M)=0$ by 2.2 . Hence $\xi(T \mid M)=0$. But then for $N \in \mathcal{I}(M)$ we have $\xi(T \mid N)=0$, contradicting (iii).

(i) $\Rightarrow$ (ii): Immediate.

(ii) $\Rightarrow$ (v): Assume (ii). If $D(T)$ is finite dimensional there is nothing to prove. Accordingly let $D(T)$ be infinite dimensional and let $E \cap N(T)$ be finite dimensional where $E \in \mathcal{I}(D(T))$. Suppose that $T_{E} \notin F_{+}$. Write $S=T J_{E}$; thus $S \in L(E, Y)$ and $\operatorname{dim} N(S)<\infty$. Since $S \notin F_{+}$there exists $M \in \mathcal{I}(E)$ such that $S \mid M$ is a precompact operator by 2.4. By assumption there exists $N \in \mathcal{I}(M)$ with $S \mid N$ relatively almost open. Then the adjoint $(S \mid N)^{\prime}$ is relatively open by 2.7 and also compact (see 
e.g. [G, III.1.11]). Therefore $(S \mid N)^{\prime}=\left(S J_{N}\right)^{\prime}$ has finite dimensional range. But $R\left(\left(S J_{N}\right)^{\prime}\right)_{\perp}=N\left(S J_{N}\right)$ is finite dimensional. Therefore

$$
\begin{gathered}
\operatorname{dim} R\left(\left(S J_{N}\right)^{\prime}\right)=\operatorname{dim} R\left(\left(S J_{N}\right)^{\prime}\right)_{\perp}^{\perp}=\operatorname{dim} N\left(\left(S J_{N}\right)^{\perp}=\right. \\
\operatorname{dim}\left(E / N\left(S J_{N}\right)\right)^{\prime}=\operatorname{dim} E / N\left(S J_{N}\right)=\infty,
\end{gathered}
$$

a contradiction. Hence (ii) $\Rightarrow$ (v).

(v) $\Rightarrow$ (vi): Assume (v) and let $T \mid M$ be injective where $M \in \mathcal{I}(D(T))$. Then $T$ is an $F_{+}$-operator. Therefore $T \mid M$ is not precompact.

(vi) $\Rightarrow$ (i): Suppose $T$ is not UO. Then by the equivalence (i) $\Rightarrow$ (iv) above, there exists $M \Rightarrow \mathcal{I}(D(T))$ such that $T \mid M$ is injective and strictly singular. In that case there exists $N \Rightarrow \mathcal{I}(M)$ for which $T \mid N$ is precompact by 2.2 , contradicting (vi).

(i) $\Rightarrow$ (vii): Assume (i). Let $M$ be any linear complement of $N(T)$ in $D(T)$; thus $D(T)=M+N(T)$ where $M \cap N(T)=0$. Then $T_{M}$ is an $F_{+}$-operator (by (i) $\Rightarrow(\mathrm{v})$ ). Hence then exist linear subspaces $E$ and $F$ of $M$ such that $M=E+F$, where $F$ is finite dimensional and $(T \mid E)^{-1}$ is continuous.

It only remains to verify that $T \mid E+N(T)$ is relatively open. We have

$$
\begin{aligned}
\gamma(T \mid E+N(T))= & \sup \{\gamma:\|T(e+n)\| \geq \gamma d(e+n, N(T)) \\
& \quad \text { for all } e \in E, n \in N(T)\} \\
= & \sup \{\gamma:\|T e\| \geq \gamma d(e, N(T)) \text { for all } e \in E\} \\
= & \sup \{\gamma:\|T e\| \geq \gamma\|e\| \text { for all } e \in E\} \\
= & \gamma(T \mid E)>0
\end{aligned}
$$

as required.

(vii) $\Rightarrow$ (i): Suppose that $T$ is not UO. Then there exists $N \in \mathcal{I}(D(T)$ ) for which $T_{M}$ is injective and precompact (by (vi) $\Rightarrow$ (i) above) clearly contradicting (vii).

(i) $\Rightarrow$ (viii): Both statements are immediate.

(viii) $\Rightarrow$ (i): It is clearly sufficient to prove the first statement. We may clearly suppose that $D(T)$ is infinite dimensional. Let $E$ be a finite codimensional subspace of $D(T)$ for which $T_{E}$ is UO. Let $M \in \mathcal{I}(D(T)$ ). Then $\Delta(T \mid M)=\Delta(T \mid E \cap M)$ by 2.3 and $\alpha(T \mid M) \geq \alpha(T \mid E \cap M)$. Consequently $\xi(T \mid M) \geq \xi(T \mid E \cap M)$. Hence $T$ is UO is by the equivalence (i) $\Rightarrow$ (iv). 
3.2. Corollary. Let $\bar{\beta}(T)<\infty$. Then the following statements are equivalent:

(i) $T$ is almost open.

(ii) $T^{\prime}$ is ubiquitously open.

(iii) $T^{\prime}$ is a $\phi_{+}$-operator.

Proof: We have $\bar{\beta}(T)=\alpha\left(T^{\prime}\right)<\infty$. Now $T$ is almost open if and only if $T^{\prime}$ is open (2.7) if and only if $T^{\prime}$ is a $\phi_{+}$-operator (see e.g. [G, IV.1.6]), if and only if $T^{\prime}$ is ubiquitously open (by Theorem 3.1).

3.3. Theorem. Let $P$ be a linear projection. Then $P$ has an infinite dimensional injective precompact restriction if and only if both $N(P)$ and $R(P)$ are infinite dimensional.

Proof: Let $M$ be an infinite dimensional subspace of $D(P)$ for which $P \mid M$ is injective and precompact. If $\operatorname{dim} N(P)<\infty$ then $R(P)$ has finite codimension in $D(P)$ and hence $M \cap R(P)$ is an infinite dimensional subspace upon which $P$ is both an isomorphism and precompact, which is impossible. Therefore $\operatorname{dim} N(P)=\infty$. On the other hand if $\operatorname{dim} R(P)<$ $\infty$ then $P$ does not have an infinite dimensional injective restriction, a contradiction. Hence necessity follows.

Conversely suppose that both $N(P)$ and $R(P)$ are infinite dimensional. Choose a linearly independent sequence $\left(a_{n}\right)$ in $R(P)$ such that $\left\|a_{n}\right\| \rightarrow 0$. Next construct a sequence $\left(b_{n}\right)$ in $N(P)$ such that $\left\|b_{n}\right\|=1$ and $\left\|b_{n}-b_{k}\right\| \geq 1(n \neq k)$ (for example, by the Gramm-Schmidt construction). Write $x_{n}=a_{n}+b_{n}$ and $E=\operatorname{sp}\left\{x_{n}\right\}$. Then $P \mid E$ is injective; indeed $e=\Sigma \alpha_{i} x_{i}, P e=0 \Rightarrow \Sigma \alpha_{i} P x_{i}=\Sigma \alpha_{i} a_{i}=0 \Rightarrow e=0$ by the linear independence of $\left\{a_{i}\right\}$. Furthermore $P X_{n}=a_{n} \rightarrow 0$ and $\left(x_{n}\right)$ has no Cauchy subsequence since $\left\|x_{n}-x_{k}\right\| \geq\left\|b_{n}-b_{k}\right\|-\left\|a_{n}-a_{k}\right\|>\frac{1}{2}$ eventually. Therefore $P \mid E$ is not an $F_{+}$-operator by $[\mathbf{C 4}, 21]$ and hence has an infinite dimensional precompact restriction [G, III.1.9].

\subsection{Theorem. The following are equivalent:}

(i) $T$ is ubiquitously open.

(ii) Either $T$ is an $F_{+}$-operator or $R(T)$ is finite dimensional.

Proof: For simplicity of notation let $D(T)=X$. Assume (i) and suppose in the first instance that $T$ is continuous. Write $X=N(T)+E$ where $N(T) \cap E=\{0\}, S=(T \mid E)^{-1}$ and $P=S T$. Then $P$ is a projection with $N(P)=N(T)$ and $R(P)=E$. Let $M \in \mathcal{I}(X)$. By 
hypothesis there exists $N \in \mathcal{I}(M)$ for which $\gamma(T \mid N)>0$. We have

$$
\begin{aligned}
\gamma(P \mid N) & =\gamma(S T \mid N)=\inf _{n \in \mathbb{N}} \frac{\|S T n\|}{\|n+N(T \mid N)\|} \geq \inf _{n \in \mathbb{N}} \frac{\gamma(S)\|T n\|}{\|n+N(T \mid N)\|} \\
& =\gamma(S) \gamma(T \mid N)>0
\end{aligned}
$$

since $\gamma(S)=\|T \mid E\|^{-1}>0$ by the continuity of $T$. Therefore $P$ is UO. By Theorem 3.3 either $\operatorname{dim} R(T)=\operatorname{dim} E<\infty$ or $\operatorname{dim} N(T)<\infty$. In the latter case $T \in F_{+}$. Hence (i) $\Rightarrow$ (ii) in the case when $T$ is continuous. Passing now to the general case when $T$ is arbitrary, we note that for any subspace $M$ of $X$,

$$
\gamma(T \mid M)>0 \Leftrightarrow \gamma\left(T G \mid G^{-1} M\right)=\gamma\left(T \mid M \circ G_{T \mid M}\right)>0
$$

by 2.6 and hence $T$ is UO if and only if $T G$ is UO. Since $T G$ is continuous, it follows from what has been proved that either $R(T)=R(T G)$ is finite dimensional or $T G \in F_{+}$. But clearly $T G \in F_{+} \Rightarrow T \in F_{+}$. Therefore (i) $\Rightarrow$ (ii).

Conversely assume (ii). If $R(T)$ is finite dimensional then clearly $T$ is UO. On the other hand if $T$ is an $F_{+}$-operator then so are all its restrictions and hence by (vii) of Theorem $3.1, T$ is UO.

3.5. Corollary. Let $X$ and $Y$ be Banach spaces and $T$ a closed operator with infinite dimensional range. Then the following are equivalent:

(i) $T$ is a $\phi_{+}$-operator.

(ii) $T$ is ubiquitously open.

Then next corollary is a uniform boundedness principle for the minimum modulus function $\gamma(T)$.

3.6. Corollary. The following statements are equivalent for an arbitrary operator $T: D(T) \subset X \rightarrow Y$ with infinite dimensional domain $D(T)$ :

(i) For each $M \in \mathcal{I}(D(T))$ we have $\sup _{N \in \mathcal{I}(M)} \gamma(T \mid N)>0$.

(ii) $\inf _{M \in \mathcal{I}(D(T))} \sup _{N \in \mathcal{I}(M)} \gamma(T \mid N)>0$.

Proof: Assume (i). Then $T$ is UO. Hence either $\operatorname{dim} R(T)<\infty$ or $T \in F_{+}$by Theorem 3.4. In the former case $\gamma(T \mid N)=\infty$ for each $N \in \mathcal{I}(N(T))$ and so (ii) holds. In the latter case we have

$$
\inf _{M \in \mathcal{I}(D(T))} \sup _{N \in \mathcal{I}(M)} \gamma(T \mid N) \geq \inf _{M} \sup _{N} \inf _{n \in N} \frac{\|T n\|}{\|n\|}=\inf _{M \in \mathcal{I}(D(T))} \tau(T \mid M) .
$$


Let $E$ be a finite codimensional subspace of $D(T)$ for which $(T \mid E)^{-1}$ exists and is continuous. Then $M \cap E$ has finite codimension in $M$ for each $M \in \mathcal{I}(D(T))$ and then $\tau(T \mid M)=\tau(T \mid M \cap E)$ by 2.3. Therefore

$$
\inf _{M \in \mathcal{I}(D(T))} \tau(T \mid M)=\inf _{M \in \mathcal{I}(E)} \tau(T \mid M)=\Delta\left((T \mid E)^{-1}\right)^{-1} .
$$

But $(T \mid E)^{-1}$ is continuous and so $\Delta\left((T \mid E)^{-1}\right)<\infty$. Hence (ii) follows.

\subsection{Corollary.}

(a) The sum of a ubiquitously open operator and a finite rank operator is ubiquitously open.

(b) The product of two ubiquitously open operators is ubiquitously open.

Proof:

(a) Immediate from the equivalence (i) $\Rightarrow$ (viii) of Theorem 3.1 .

(b) Combine Theorem 3.4 with 2.5 .

3.8. Corollary. If $T$ is ubiquitously open then so is $\hat{T}$.

According to Corollary 3.8 the continuous linear maps between coincomparable Banach spaces [GO], except those of finite rank, are all not UO. The situation is similar when the two spaces are totally incomparable Banach spaces (cf. $[\mathbf{R}]$ ); indeed such maps are strictly singular.

3.9. Proposition. Let $T$ be partially open. Then $\hat{T}$ is partially open.

Proof: Let $E$ be a finite codimensional subspace of $D(T)$ for which $T_{E}$ is open. Write $Q=Q_{N(T)}^{X}$. We have

$$
\gamma(\hat{T} \mid Q E)=\inf _{e \in E} \frac{\|\hat{T} Q e\|}{\|Q e\|} \geq \inf _{e \in E} \frac{\|T e\|}{\left\|e+N\left(T_{E}\right)\right\|}=\gamma\left(T_{E}\right)>0 .
$$

Since $Q E$ is finite codimensional in $Q(D(T))=D(\hat{T}), \hat{T}$ is partially open.

3.10. Proposition. Let $X$ and $Y$ be Banach spaces and $T$ a closed operator. Then $T$ is partially open if and only if it is open. 
Proof: Recall that $T$ if $R O$ if and only if $T G_{T}$ is $R O$ (2.6). Hence also $T$ is $P R O$ if and only if $T G_{T}$ is $P R O$. Furthermore $X_{T}$ is a Banach space. Consequently we may suppose that $T$ is bounded. Let $T$ be partially open. Then $\hat{T}$ is partially open by Proposition 3.9. Hence $\hat{T}$ is an isomorphism by 2.1 . Therefore $T$ is open. The converse statement is trivial.

3.11. Theorem. Let $X$ and $Y$ be Banach spaces and $T: D(T) \subset$ $X \rightarrow Y$ a closed operator. Then the following statements are equivalent:

(i) $T$ is either a $\phi_{+}$-operator or a bounded finite rank operator.

(ii) For each linear subspace $M$ of $D(T)$ we have $\overline{T M} \subset T \bar{M}$.

Proof: Assume (i). Then $T$ is UO by Theorem 3.4. Let $M$ be an arbitrary subspace of $D(T)$. By Theorem $3.1 T \mid \bar{M}$ is partially open and hence open by Proposition 3.10. Therefore $T(\bar{M})$ is closed by [G, IV.1.6]. Hence (i) $\Rightarrow$ (ii).

Conversely, assume (ii). Let $R(T)$ be infinite dimensional. Suppose $M \in \mathcal{I}(D(T))$ where $M \cap N(T)=(0)$. If $T \mid M$ is compact, then by the density invariant result $\bar{\Gamma}_{0}(T \mid M)=\bar{\Gamma}_{0}(T \mid \bar{M})[\mathbf{C 1 1}, 3.7], T \mid \bar{M}$ is also compact, while $T(\bar{M})$ contains the infinite dimensional Banach space $\overline{T M}$, which is impossible. Hence $T$ is UO by Theorem 3.1 and consequently $T$ is a $\phi_{+}$-operator by Corollary 3.5. Since closed finite rank operators are bounded, the conclusion (i) now follows.

\section{References}

[C1] R. W. Cross, Some continuity properties of linear transformations in normed spaces, Glasgow Math. J. 30 (1988), 243-247.

[C2] R. W. CRoss, Properties of some norm related functions of unbounded linear operators, Math. Z. 199 (1988), 285-302.

[C3] R. W. CRoss, Unbounded strictly singular operators, Proc. Netherlands Acad. Sc. Series A - Indag. Math. 91, no. 3 (1988), 245-248.

[C4] R. W. CRoss, Linear operators of upper semi-Fredholm type in normed spaces, Portugal. Math. 47, no.1 (1990), 61-79.

[C5] R. W. CRoss, The essential state diagram of a linear operator, Ann. Acad. Sci. Fenn. Ser. A.I. Math. 15 (1990), 283-291.

[C6] R. W. CROss, On the perturbation of unbounded linear operators with topologically complemented ranges, J. Funct. Anal. 92, no. 2 (1990), 468-473. 
[C7] R. W. CRoss, Linear transformations of Tauberian type in normed spaces, Note Math. X, Suppl. no.1, volume dedicated to the memory of Professor Gottfried M. Kothe (1990), 193-203.

[C8] R. W. Cross, On a theorem Kalton and Wilansky concerning Tauberian operators, J. Math. Anal. Appl. 171, no.1 (1992), 156-170.

[C9] R. W. CRoss, A characterisation of almost reflexive normed spaces, Proc. R. Ir. Acad. 92A, no.2 (1992), 225-228.

[C10] R. W. CRoss, $F_{+}$-operators are Tauberian, Quaestiones Math. 16, no. 2 (1993), 129-132.

[C11] R. W. CRoss, On certain densely invariant quantities of linear operators, Preprint.

[CL1] R. W. Cross and L. E. Labuschagne, Partially continuous and semicontinuous linear operators in normed spaces, Expo. Math. 7 (1989), 189-191.

[CL2] R. W. Cross and L. E. Labuschagne, Characterisations of operators of lower semi-Fredholm type in normed spaces, Quaesiones Math. 15, no. 2 (1992), 151-173.

[D] D. van Dulst, On strictly singular operators, Compositio Math. 23, no. 2 (1971), 169-183.

[G] S. Goldberg, "Unbounded linear operators," McGraw-Hill, New York, 1966.

[GO] M. GonzÁlez and V. M. Onieva, On incomparibility of Banach spaces, Math. Z. 192 (1986), 581-585.

[K] T. Kato, Perturbation theory for nullity, deficiency and other quantities of linear operator, J. d'Analyse Math. 6 (1958), 273-322.

[L1] L. E. LABuschagne, On the minimum modulus of an arbitrary linear operator, Quaestiones Math. 14 (1991), 77-91.

[L2] L. E. LABUSChaGne, The perturbation of relatively open operators with reduced index, Math. Proc. Cambridge Phil. Soc. 112 (1992), 385-402.

[L3] L. E. Labuschagne, Certain norm related quantities of unbounded linear operators, Math. Nachr. 157 (1992), 137-162.

[MS] R. Mennicken and B. Sagraloff, Characterizations of nearly-openess, J. Reine. Angew. Math. 313 (1980), 105-115. 
[R] H. P. Rosenthal, On totally incomparable Banach spaces, $J$. Funct. Anal. 4 (1969), 167-175.

[S] M. SCHECHTER, Quantities related to strictly singular operators, Indiana Univ. Math. J. 21 (1972), 1061-1071.

\author{
Department of Mathematics \\ University of Cape Town \\ Rondebosch 7700 \\ SOUTH AFRICA
}

Primera versió rebuda el 21 de Juny de 1993, darrera versió rebuda el 31 de Gener de 1994 